as $(1 / V) \Psi_{\mathbf{h}} \sum_{\mathbf{k}} F_{\mathrm{h}-\mathrm{k}} F_{\mathrm{k}}$. However, this is not correct because the operation $\psi * \rho^{2}$, which is equivalent to $(1 / V) \Psi_{\mathbf{h}} \sum_{\mathbf{k}} F_{\mathbf{h}-\mathbf{k}} F_{\mathbf{k}}$, exaggerates errors in the current densities. The same question points to the current use of the tangent formula (for a review see Woolfson, 1987). As previously shown (Barrett \& Zwick, 1971), it is possible to obtain from Sayre's equation

$$
\tan \varphi_{\mathbf{h}}=\frac{\sum_{\mathbf{k}}\left|F_{\mathbf{h}-\mathbf{k}} F_{\mathbf{k}}\right| \sin \left(\varphi_{\mathbf{h}-\mathbf{k}}+\varphi_{\mathbf{k}}\right)}{\sum_{\mathbf{k}}\left|F_{\mathbf{h}-\mathbf{k}} F_{\mathbf{k}}\right| \cos \left(\varphi_{\mathbf{h}-\mathbf{k}}+\varphi_{\mathbf{k}}\right)},
$$

which is essentially the same as the tangent formula (Karle \& Hauptman, 1956). In the light of the argument given above, the phases calculated from this equation are not more correct than the current ones but new more correct phases should be obtained through some process that equalizes the current phases with the calculated ones.

\section{References}

Barrett, A. N. \& Zwick, M. (1971). Acta Cryst. A27, 6-11. Bernstein, F. C., Koetzle, T. F., Williams, G. J: B., Meyer, E. F. JR, Brice, M. D., Rodgers, J. R., Kennard, O., Shimanovchi, T. \& Tasumi, M. (1977). J. Mol. Biol. 112, 535-542.

Cutfield, J. F., Dodson, E. J., Dodson, G. G., Hodgkin, D. C., Isaacs, N. W., Sakabe, K. \& SaKabe, N. (1975). Acta Cryst. A31, S21.

Debaerdemaeker, T., Tate, C. \& Woolfson, M. M. (1988). Acta Cryst. A44, 353-357.
Gull, S. F. \& DANIEL, G. J. (1978). Nature (London), 272, 686-690.

Hall, S. R. \& StewarT, J. M. (1990). Editors. XTAL3.0 Reference Manual. Univs. of Western Australia, Australia, and Maryland, USA.

HeNDRICKSON, W. A. (1985). Mathods Enzymol. 115, 252-270.

HENDRICKSON, W. A. \& TEETER, M. M. (1981). Nature (London), 290, 109-113.

Hestenes, M. R. (1969). J. Optim. Theor. Appl. 4, 303-320.

HOPPE, W. (1963). Z. Kristallogr. 118, 121-126.

Hoppe, W. \& Gassman, J. (1964). Ber. Bunsenges. Phys. Chem. 68, 808-817.

ICHIKAWA, K. (1975). SICE Trans. 11, 180-186. (In Japanese.)

Karle, J. \& Hauptman, H. (1956). Acta Cryst. 9, 635-651.

Kirkpatrick, S., Gelatt, C. G. JR \& Vecchi, M. P. (1983). Science, 220, 671-680.

Krabbendam, H. \& Kroon, J. (1971). Acta Cryst. A27, 48-53.

Livesey, A. L. \& Skilling, J. (1985). Acta Cryst. A41, 113-122.

MaIn, P. (1990). Acta Cryst. A46, 372-377.

Navaza, J. (1986). Acta Cryst. A42, 212-223.

Sato, T. (1984). Acta Cryst. C40, 880-882.

Sato, T. (1992). Acta Cryst. A48, 842-850.

SATO, T. (1994). In preparation.

SAYRE, D. (1952). Acta Cryst. 5, 60-65.

SAYre, D. (1972). Acta Cryst. A28, 210-212.

SAYRE, D. (1974). Acta Cryst. A30, 180-184.

SAYRE, D. (1975). Crystallographic Computing Techniques, edited by M. F. C. LADD \& R. A. PAlmer, pp. 271-286. New York: Plenum Press.

Shiono, M. \& Woolfson, M. M. (1991). Acta Cryst. A47, 526-533.

Wilkins, S. W., Varghese, J. N. \& Lehmann, M. S. (1983). Acta Cryst. A39, 47-60.

Woolfson, M. M. (1987). Acta Cryst. A43, 593-612.

Woolfson, M. M. \& YAO, J.-X. (1990). Acta Cryst. A46, 409413.

ZhaNG, K. Y. J. \& MaIN, P. (1990). Acta Cryst. A46, 377-381.

Acta Cryst. (1994). A50, 383-391

\title{
Novel Treatment of the Experimental Data in the Application of the Maximum-Entropy Method to the Determination of the Electron-Density Distribution from X-ray Experiments
}

\author{
By R. Y. DE VRIES, W. J. BRiels and D. FeIL \\ Chemical Physics Laboratory, University of Twente, PO Box 217, 7500 AE Enschede, The Netherlands
}

(Received 6 September 1993; accepted 17 November 1993)

\begin{abstract}
The maximum-entropy method (MEM) has been tested on a limited set of noisy Fourier data from a known electron-density distribution (EDD). It is shown that maximizing the entropy of the EDD under the usual condition of fitting the variance of the data set does not necessarily lead to a satisfactory error distribution of the calculated reflections. The MEM property of producing the flattest EDD consistent with the data causes the calculated values of strong reflections to deviate systematically as much

as possible from their measured values. Calculated values of strong reflections are usually smaller than their measured values. The use of a novel constraint on the entropy maximization greatly improves the form of the error distribution and also the calculated EDD.

\section{Introduction}

The common method of extracting the EDD from an incomplete and noisy data set is to fit the data to a ISSN 0108-7673 C1994 
model that depends on adjustable parameters. One of the reasons a model is used is to reduce the effect of noise in the measured reflections on the resulting EDD. The global characteristics of the resulting EDD are expected to be correct since these are incorporated in the model. However, if there are subtle features in the EDD that cannot be accommodated by the model, these features will either get lost or show up in distorted form in the EDD. Several programs [e.g. POP (Craven, Weber \& He, 1987), MOLLY (Hansen \& Coppens, 1978) and LSEXP (Hirshfeld, 1971, 1977)] exist to model the data. They all use the least-squares method to obtain the fit parameters but their models differ in functional form and in number of adjustable parameters. EDDs obtained with these programs are often slightly different from each other (Baert, Coppens, Stevens \& Devos, 1982; Lecomte, 1991). This raises the question of the best estimate of the EDD given a certain data set.

The EDD is a positive and additive quantity. In this case, the principle of maximum entropy states that, out of all the EDDs consistent with the data, we should choose the one that maximizes the Shannon-Jaynes entropy (Jaynes, 1983), defined in the next section.

The data are a set of $M$ measured reflections. The system studied is a centrosymmetric crystal in which the phase problem is assumed to be solved. The experimental structure factors are denoted $D_{h k l}$. The structure factors corresponding with the desired EDD are denoted $F_{h k l}$. With the assumption that the experimental errors $\left(\sigma_{h k l}\right)$ are independent and that $M$ is large enough, the quantity

$$
C=\sum_{h, k, l}\left(F_{h k l}-D_{h k l}\right)^{2} / \sigma_{h k l}^{2}
$$

will satisfy

$$
C=M \text {. }
$$

This is the only information provided by the experiment and serves as a constraint in the maximization of the entropy.

The unconstrained maximum of the entropy is given by a flat EDD. This means that, if we do not have any data apart from a knowledge of the total number of electrons per unit cell, the best estimate we can make is a flat EDD, any other estimate being based on some sort of information. If we do have data, it is still possible that the unconstrained maximum satisfies $C \leq M$, which means the data are too noisy for any information to be extracted. Otherwise, the solution lies on the boundary, $C=M$.

For the resulting EDD, the quantities

$$
\left(\left|F_{h k l}\right|-\left|D_{h k l}\right|\right) / \sigma_{h k l}
$$

may be calculated and presented in a frequency histogram. This histogram should be nearly Gaussian from a statistical point of view. With the application of the usual maximum-entropy method in EDD studies, this is not the case (Jauch \& Palmer, 1993), with serious consequences for the EDD. We anlyse the cause of this artefact and suggest a solution to it in $\S 4$.

In $\$ 2$, we describe some computational aspects of the program that was written to perform the entropy maximization. In $\S 3$, we present the results of an application of the method to the Fourier components of a hypothetical crystal with a known EDD. We have calculated those Fourier components that would be measured in a standard X-ray experiment and added noise. After presenting our modification of the method in $\S 4$, we apply the new method to the same Fourier components. Finally, in $\$ 5$, we apply both methods to the Pendellösung data for silicon measured by Saka \& Kato (1986). We compare our results with those of a previous application of the MEM to this data by Sakata \& Sato (1990).

\section{Description of the algorithm}

It is our aim to maximize the Shannon entropy $\bar{S}(f)$, defined by

$$
\begin{gathered}
\bar{S}(f)=-\sum_{i=1}^{N_{1}} \sum_{j=1}^{N_{2}} \sum_{m=1}^{N_{3}} p_{i j m} \ln p_{i j m}, \\
p_{i j m}=\left[1 / \sum(f)\right] f_{i j m}, \\
\sum(f)=\sum_{i=1}^{N_{1}} \sum_{j=1}^{N_{2}} \sum_{m=1}^{N_{3}} f_{i j m},
\end{gathered}
$$

where the electron density $f(\mathbf{r})$ in the crystallographic unit cell is represented by its values $f_{i j m}$ on a threedimensional grid of $N_{1} \times N_{2} \times N_{3}$ points. The maximization has to be done under the constraints

$$
\begin{gathered}
f_{i j m} \geq 0, \\
C(f)=\sum_{h, k, l}\left\{\left[F_{h k l}(f)-D_{h k l}\right] / \sigma_{h k l}\right\}^{2}=M, \\
T(f)=\left(V_{\text {cell }} / N_{1} N_{2} N_{3}\right) \sum_{i, j, m} f_{i j m}=N .
\end{gathered}
$$

We make sure that the first constraint is satisfied by using $\ln f_{i j m}$ as our basic variables instead of $f_{i j m}$. In the second constraint (8), the sum runs over all measured unique reflections. $F_{h k l}(f)$ and $D_{h k l}$ are the calculated and measured structure factors, respectively; $M$ is the number of measured unique reflections. It is useful to expand the sum in $C(f)$ so as to include all symmetry-related reflections in order to be able to make use of fast-Fourier-transform algorithms. The value of $C(f)$ is kept the same by multiplying all the variances $\sigma_{h k l}^{2}$ by the total number of equivalent reflections $F_{h k l}$. The triple sum in $T(f)$ 
is a shorthand notation for the sum over all grid points. $N$ is the total number of electrons per unit cell.

The usual way to incorporate the constraints into the maximization is to introduce a Lagrange multiplier for each constraint and optimize

$$
\bar{Q}(f)=\bar{S}(f)-\lambda C(f)-\mu T(f) .
$$

One then optimizes $\bar{Q}(f)$ unconditionally for given values of $\lambda$ and $\mu$ and changes $\lambda$ and $\mu$ until the constraints on $C(f)$ and $T(f)$ are satisfied. Here, we adopt a method developed by Skilling \& Bryan (1984), who maximize

$$
\begin{gathered}
Q(f)=\alpha S(f)-C(f), \\
S(f)=-\sum_{i, j, m} f_{i j m} \ln \left(f_{i j m} / A\right)-\sum(f),
\end{gathered}
$$

where $\alpha$ is chosen such that (8) is satisfied and $A$ such that (9) is satisfied. It is not difficult to demonstrate that (10) and (11) have the same solutions. Equation (11), however, has several computational advantages.

In the actual calculations, we choose $A$ and optimize $Q(f)$ under the constraint (8). We then vary $A$ until (9) is satisfied. The optimization of $Q(f)$ consists of two parts. In the first part, we make sure that we gradually approach the surface $C(f)=M$. Once we are on this surface, we optimize $Q(f)$ while at the same time we choose $\alpha$ such that we stay on the surface $C(f)=M$. The constrained maximum is obtained when the vectors $\partial S / \partial f_{i j m}$ and $\partial C / \partial f_{i j m}$ are parallel. We check this by calculating

$$
\begin{aligned}
\delta= & \left(\sum_{i j m}\left\{\frac{\partial S}{\partial f_{i j m}} /\left[\sum_{a b c}\left(\frac{\partial S}{\partial f_{a b c}}\right)^{2}\right]^{1 / 2}\right\}\right. \\
& \left.-\sum_{i j m}\left\{\frac{\partial C}{\partial f_{i j m}} /\left[\sum_{a b c}\left(\frac{\partial C}{\partial f_{a b c}}\right)^{2}\right]^{1 / 2}\right\}\right)^{2} .
\end{aligned}
$$

When $\delta<10^{-3}$, we assume that our calculation has converged. A detailed description of the program is given in Appendices $A$ and $B$.

In Figs. $1(a)$ and $(b)$, we have visualized the value of the constraint $C(f)$ and the value of the entropy $\bar{S}(f)$ from the run on the silicon data $(\S 5)$.

\section{Test on a hypothetical water crystal}

To test the maximum-entropy method, a set of structure factors was generated from a known density. This density was constructed in three steps. First, the density of one water dimer was calculated from an $a b$ initio molecular wavefunction using a $6-31 G^{* *}$ basis set. Two water dimers were put into a centrosymmetric unit cell (Fig. 2) with dimensions 6 $\times 6 \times 3 \AA$ and $\alpha=\beta=\gamma=90^{\circ}$ (space group $P 2 / m$; unique axis $c$ ). Next, the EDD was partitioned into atomic contributions and thermally smeared with the assumption of independent atomic vibrations, as described by Bruning \& Feil (1992). Isotropic temperature factors for the atoms at $100 \mathrm{~K}$ were taken from Eriksson \& Hermansson (1983). The resulting reflections were finally transformed into our EDD by Fourier summation of all the reflections with $(\sin \theta) / \lambda<5 \AA^{-1}$. The result is plotted in Fig. 3 . From now on, we call this EDD our reference EDD.

Experimentally, when molybdenum radiation is used, one can only measure reflections for which $(\sin \theta) / \lambda<1.4 \AA^{-1}$. As is well known, a direct Fourier transformation of this limited set of reflections usually yields rather bad EDDs, even when the reflections can be measured exactly. In Fig. 4, we present the result of such a direct Fourier transformation; reflections which would have been considered insignificant in a real experiment have been

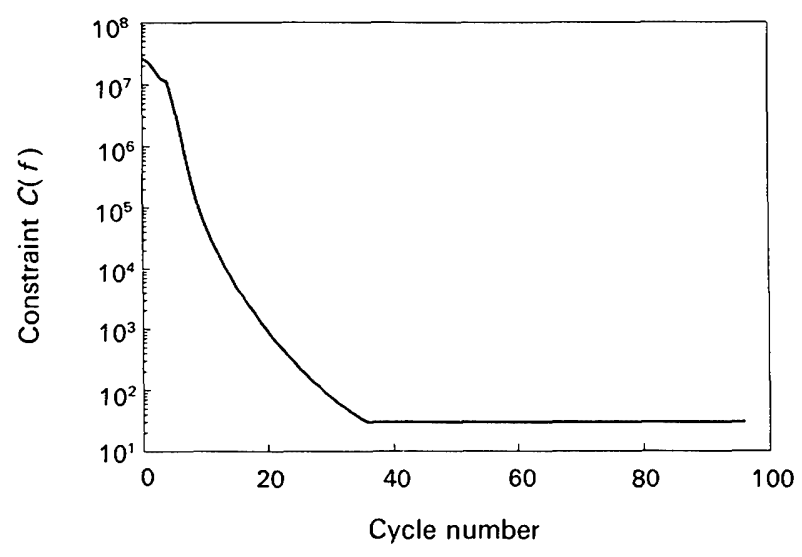

(a)

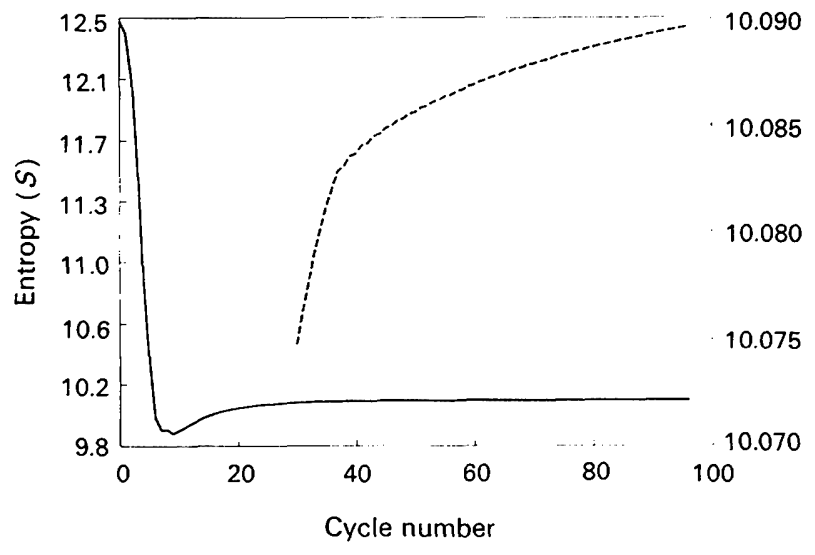

(b)

Fig. 1. Values of some quantities during a typical run of the program. (a) $C(f)$. (b) $\bar{S}(f)$. The dashed line in $(b)$ is the same curve but on a different scale (right $y$ axis). 
omitted. One easily recognizes the oscillations introduced by the abrupt truncation of the Fourier summation. The MEM does not suffer from this problem because it operates in real space; it effectively extrapolates the set of measured reflections in a smooth way. In Fig. 5, we have plotted the results of an application of the MEM with a $64 \times 64 \times 32$ grid to the exact reflections for which $(\sin \theta) / \lambda<1.4 \AA^{-1}$. In order to make the predicted reflections equal to the exactly measured reflections, we have taken nearly all the $\sigma_{h k l}$ values to be equal to 1 (for a few strong low-order reflections $\sigma_{h k l}=0.1$ ) and made $C \simeq 0.2$. Fig. 5 shows, rather spectacularly, the great power of the MEM as a tool to diminish truncation effects. The difference between the MEM density and the reference EDD is given in Fig. 6. The main differences occur at the nuclei of the $\mathrm{O}$ atoms.

In a real experiment, all reflections are measured with a certain error. To simulate the experimental

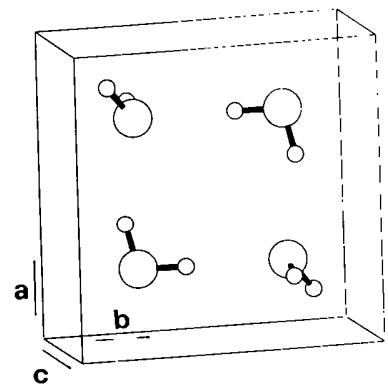

Fig. 2. The orientation of the water dimers in the unit cell. The nuclei of the lower-left water molecule and the $\mathrm{O}$ atom of the lower-right molecule are in the mirror plane $z=\frac{1}{2}$. The upper water dimer is added to make the crystal centrosymmetric.

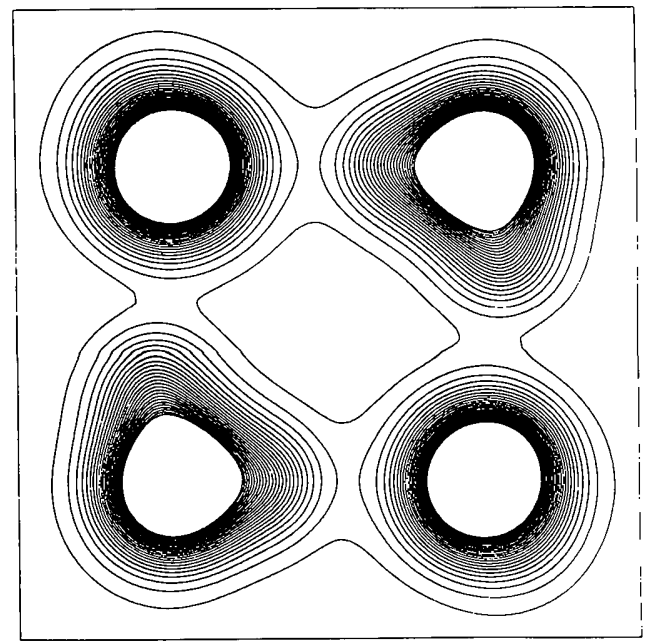

Fig. 3. The reference EDD in the $z=\frac{1}{2}$ plane, calculated from a Fourier summation of all the reflections for which $(\sin \theta) / \lambda<$ $5 \AA^{-1}$. The contours are at intervals of $0.1 \mathrm{e} \AA^{-3}$. situation, we used reflections for which $(\sin \theta) / \lambda<$ $1.4 \AA^{-1}$ and added Gaussian noise to them such that low-order reflections had an error of $1 \%$ of their absolute value (including an assumed background) and the high-order reflections had an error of $10 \%$. Reflections for which the amplitude was less than $3 \sigma$ were omitted since the phase of the reflections cannot be determined with $100 \%$ certainty (these are the reflections that we referred to in the preceding paragraph). This resulted in 1752 unique reflections.

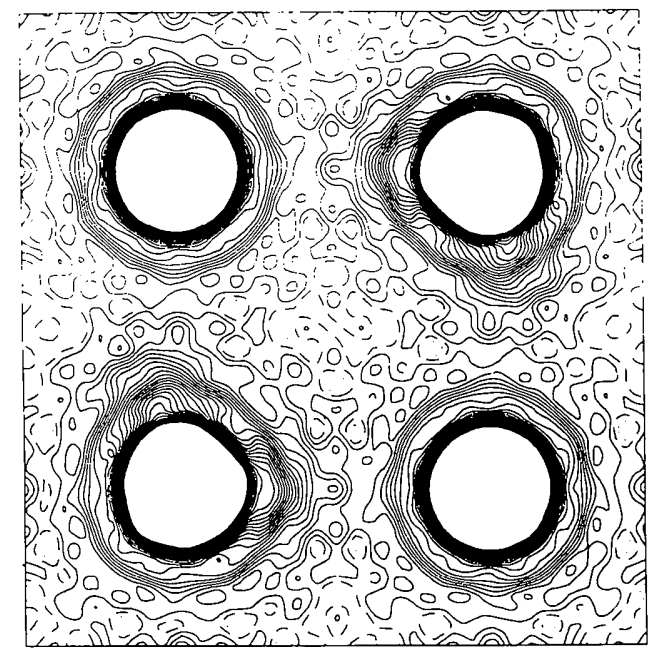

Fig. 4. The EDD resulting from a direct Fourier transformation of all the (exact) reflections calculated from the hypothetical waterdimer crystal $\left[(\sin \theta) / \lambda<1.4 \AA^{-1}\right]$. Reflections that would have been considered insignificant in a real experiment were omitted. Positive contours are drawn as solid lines, zero contours as dash-dotted lines and negative contours as dotted lines. The contours are at intervals of $0.1 \mathrm{e} \AA^{-3}$.

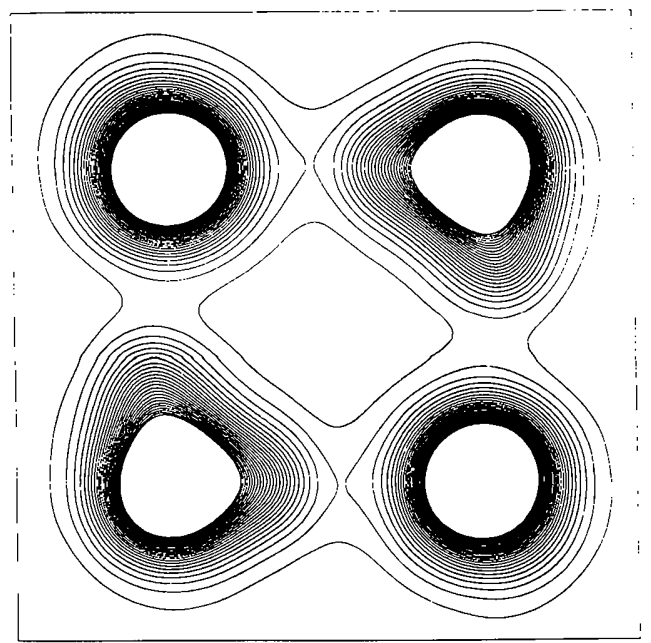

Fig. 5. The EDD calculated with the MEM from the reflections that were used to calculate the EDD of Fig. 4. The contours are at intervals of $0.1 \mathrm{e} \AA^{-3}$. 
In Fig. 7, we have plotted the EDD obtained from a MEM calculation with the data described above. Comparing this density with the reference EDD given in Fig. 3, we see that the agreement between the two is very poor. The MEM solution is little better than the direct Fourier transformation given in Fig. 4.

\section{Weighting scheme}

In this section, we investigate the reason for the poor result obtained with the MEM applied to noisy data. In constructing the 'experimental' reflections, we

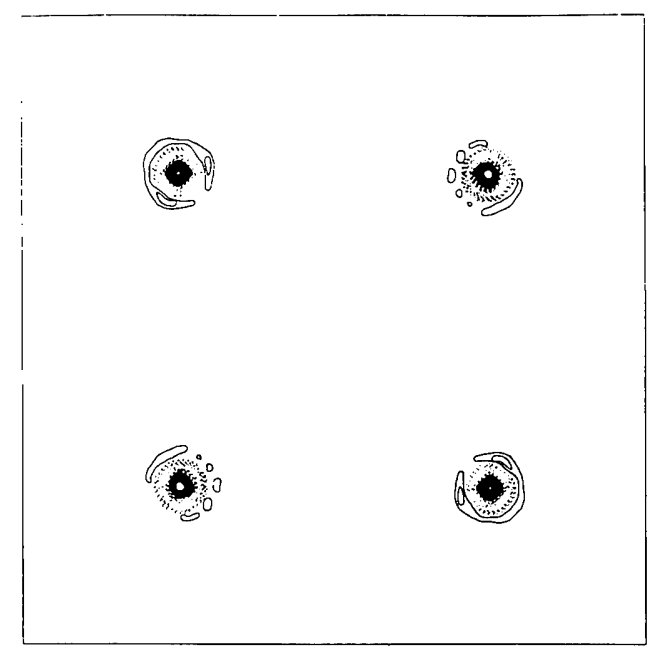

Fig. 6. The difference between the EDD obtained with the MEM (Fig. 5) and the reference density (Fig. 3). The zero contour has been omitted. The contours are at intervals of $0.1 \mathrm{e} \AA^{-3}$.

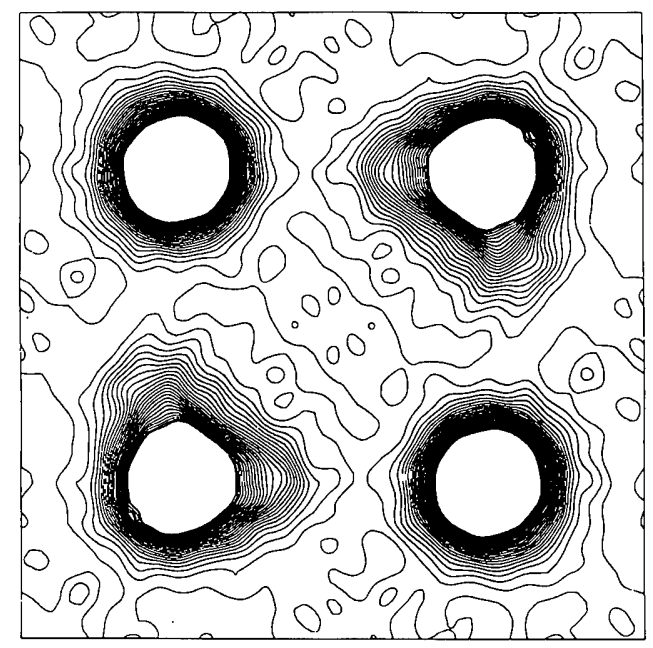

Fig. 7. The EDD calculated with the MEM from the reflections that were used to calculate the EDD of Fig. 4. Noise was added to these reflections. The contours are at intervals of $0.1 \mathrm{e} \AA^{-3}$. have assumed Gaussian noise. We therefore want the differences between the predicted and the measured structure factors to have a Gaussian distribution also. A histogram of this distribution is given in Fig. 8 , showing that the resulting distribution is far from Gaussian. In fact, a few strong low-order reflections tend to deviate by many (up to 18.8) $\sigma$ from their measured values (these are not shown in Fig. 8). As a result, the weaker high-order reflections have to be exactly equal to their measured values. By optimizing the entropy subject to $C=M$, we have constrained the variance of the resulting distribution but not its shape.

In order to gain more insight into the cause of our problem, we have calculated the entropy of a single reflection. This we have done by taking one structure factor of given wavelength and amplitude, adding $F(000)$ and calculating the entropy of the corresponding EDD. First, we have found that the result is independent of the wavelength of the structure factor. The dependence on its amplitude is given in Fig. 9. Of course, the entropy of the total EDD is not a sum of the entropies of the individual reflections but nevertheless we think that Fig. 9 gives an approximate impression of what happens to the entropy when the amplitude of one single reflection is changed. From Fig. 9, we see that the entropy decreases almost quadratically with the amplitude of the structure factor. As a result, the increase of the entropy will be much larger when a large-amplitude reflection is reduced in value than when a small amplitude reflection is reduced in value. This is exactly what happens in the optimization.

We suggest that the problems mentioned in the last paragraph can be solved by the use of a

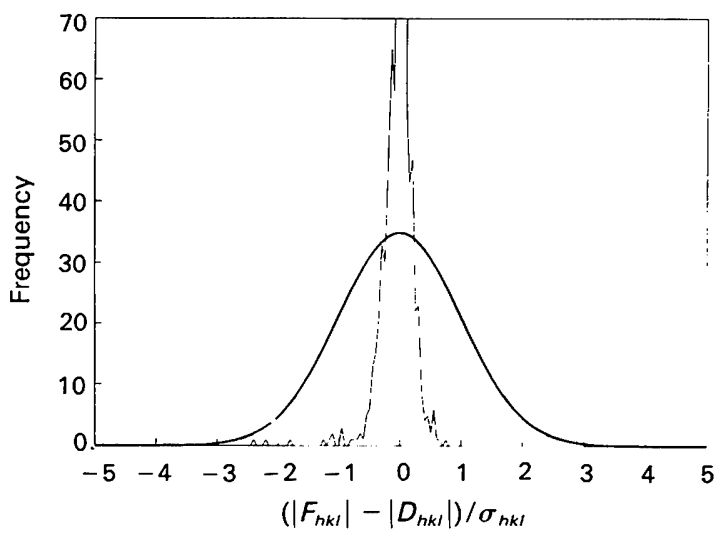

Fig. 8. Histogram of the values $\left(\left|F_{h k l}\right|-\left|D_{h k l}\right|\right) / \sigma_{h k l}$ at intervals of 0.05 from the MEM calculation. The maximum of the peak is at the interval $[-0.025,0.025]$. The height of the peak is 777 . Nine reflections, with $\left(\left|F_{h k l}\right|-\left|D_{h k l}\right|\right) / \sigma_{h k l}$ values of $-18.8,-18.75$, $-18.25,-14.90,-13.60,-10.70,-7.85,-6.40$ and -5.65 , are not shown. The thick solid line is the exact Gaussian distribution. 
weighting scheme in the constraint $C$. Therefore, we have maximized the entropy subject to

$$
K(f)=\sum_{h, k, l} w_{h k l}\left[\left(F_{h k l}-D_{h k l}\right)^{2} / \sigma_{h k l}^{2}\right]=P .
$$

The value of $w_{h k l}$ has to be large for strong low-order reflections so that they do not deviate much from their measured values. After some practice, it was found that choosing $w_{h k l}$ equal to the inverse of the length of the reciprocal-lattice vector to the fourth power,

$$
w_{h k l}=1 /|\mathbf{G}|^{4},
$$

where $\mathbf{G}=h \mathbf{a}^{*}+k \mathbf{b}^{*}+l \mathbf{c}^{*}$, gives the best results.

The value of $P$ can be chosen in several ways. One choice might be $P=\sum w_{h k l}$. Another possibility is to choose $P$ such that $C=M$. Because $w_{h k l}$ and $\left(F_{h k l}-D_{h k l}\right)^{2} / \sigma_{h k l}^{2}$ are not independent, these choices are not equivalent. We have chosen the latter possibility, i.e. $P$ such that $C=M$.

The EDD resulting from this optimization is given in Fig. 10. The map resembles the reference EDD quite well although the contour level of 0.1 e $\AA^{-3}$ is not as smooth as might be expected. This may be caused by a remaining tendency of the MEM to lower the absolute values of the strong reflections. The corresponding error distribution is presented in Fig. 11. This distribution is nearly Gaussian and there are no extremes as in the histogram presented in Fig. 8.

\section{Silicon}

Accurate Pendellösung data on silicon have been measured by Saka \& Kato (1986). The MEM has been applied to these data by Sakata \& Sato (1990). Using our program, we have calculated the EDD of silicon (on a $64 \times 64 \times 64$ grid) with and without the

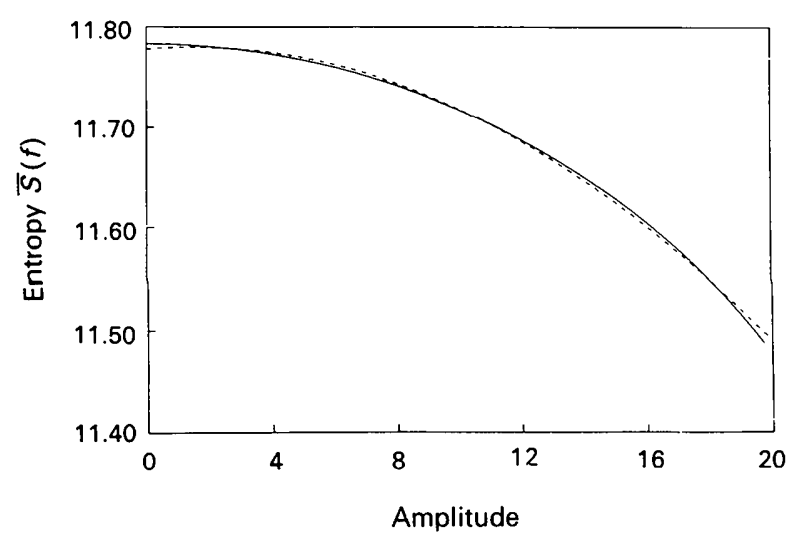

Fig. 9. The entropy of a reflection (together with its Friedel-related reflection) as a function of its amplitude. The $F(000)$ reflection has been added to avoid negative densities. The dashed line is a polynomial fit of order 2 . use of the weighting scheme proposed in the preceding section. Of course, the calculation without the use of the weighting scheme gives the same EDD as that obtained by Sakata \& Sato (1990). The EDD obtained with the use of the weighting scheme was almost exactly equal to the EDD obtained by Sakata \& Sato (1990).

The reason for this is that the error in the measured reflections is very small. With an error of zero on each reflection, the use of the weighting scheme has no effect: both methods will give the same EDD. So, when the error is very small, not much difference between the methods is to be expected. The error distributions of the methods are given in Figs. 12(a) and $(b)$. The entropy maximization in which a weighting scheme was used in the constraint gives a

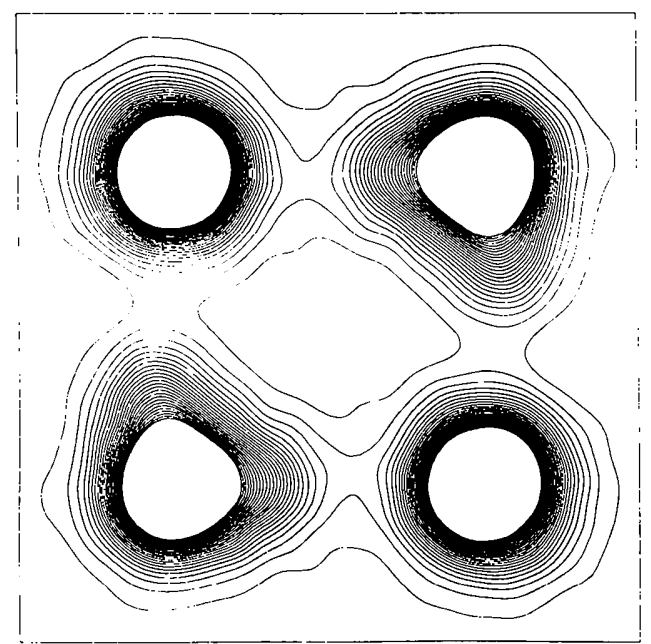

Fig. 10. The EDD calculated with the MEM using a weighting scheme in the constraint $C(f)$. The contours are at intervals of 0.1 e $\AA^{-3}$.

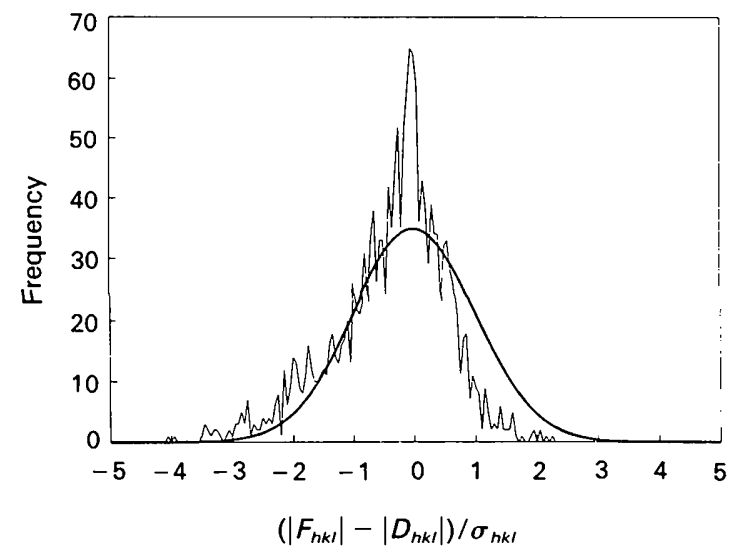

Fig. 11. Histogram of the values of $\left(\left|F_{h k l}\right|-\left|D_{h k l}\right|\right) / \sigma_{h k l}$ at intervals of 0.05 from the MEM calculation using a weighting scheme in the constraint $C(f)$. The thick solid line is the exact Gaussian distribution. 
slightly better error distribution but, since we have only 30 reflections, the statistics do not allow us to draw definite conclusions.

\section{Concluding remarks}

From the calculations on the hypothetical waterdimer crystal, we can conclude that the MEM handles the series-termination effect very well. When the data are noisy, calculated values of strong reflections from the resulting maximum-entropy EDD show large deviations from the measured values. Of all reflections, the strong reflections cause the largest decrease of entropy from its maximum, which is obtained with the flat EDD. The MEM will therefore always try to lower the absolute values of these strong reflections within the limits of the constraints. This contradicts the assumed randomness in the errors of the reflections and leads to a bad EDD. When maximizing the entropy subject to $C=M$, we are only fitting the variance of the distribution, we do not put any constraint on its shape.

The use of an appropriate weighting scheme partially solves this problem. It leads to an improved

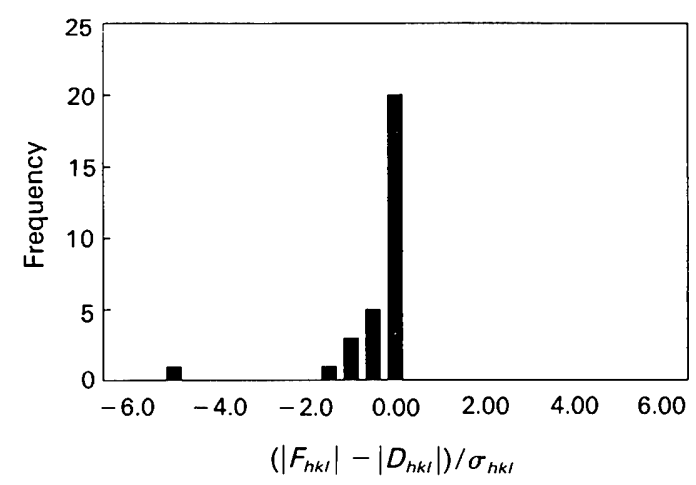

(a)

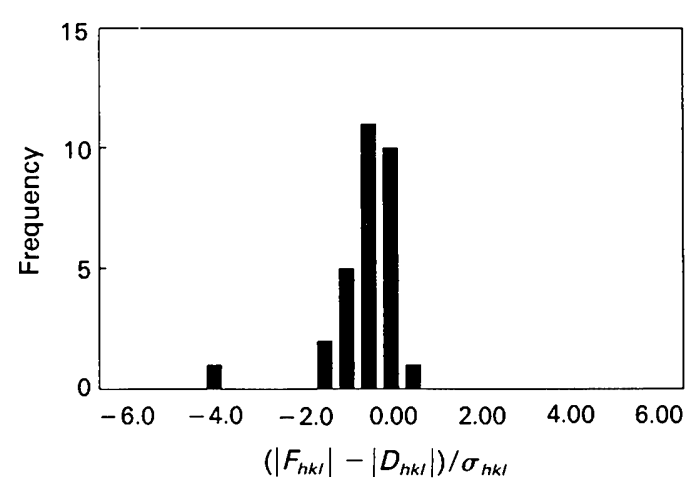

(b)

Fig. 12. Error-distribution histogram from the MEM calculation of the silicon data at intervals of 0.5. (a) No weighting scheme. (b) Weighting scheme.
EDD because it prevents the strong reflections deviating much from their measured values.

\section{APPENDIX $A$}

We now describe the optimization algorithm in some detail. We consider the densities $f_{i j m}$ as the components of an $N_{1} \times N_{2} \times N_{3}$-dimensional vector. At every step of the optimization, we choose a search direction in the space spanned by the vectors $\mathbf{e}^{1}, \mathbf{e}^{2}$ and $\mathbf{e}^{3}$, with components

$$
\begin{aligned}
& e_{i j m}^{1}=\frac{\partial S}{\partial \ln f_{i j m}}, \\
& e_{i j m}^{2}=\frac{\partial C}{\partial \ln f_{i j m}}, \\
& e_{i j m}^{3}=f_{i j m} \sum_{i^{\prime} j^{\prime}, m^{\prime}} \frac{\partial^{2} C}{\partial f_{i j m} \partial f_{i^{\prime} j^{\prime} m^{\prime}}}\left(\alpha_{1} e_{i^{\prime} j^{\prime} m^{\prime}}^{1}-\alpha_{2} e_{i^{\prime} j^{\prime} m^{\prime}}^{2}\right),
\end{aligned}
$$

where $\quad \alpha_{1}=\left[\sum\left(\partial S / \partial \ln f_{i j m}\right)^{2} / f_{i j m}\right]^{-1 / 2}$ and $\alpha_{2}=$ $\left[\sum\left(\partial C / \partial \ln f_{i j m}\right)^{2} / f_{i j m}\right]^{-1 / 2}$. Note that, in the definitions of $\mathbf{e}^{1}$ and $\mathbf{e}^{2}$, we explicitly use $\ln f_{i j m}$ as the basic variables. This does not hold true for $\mathbf{e}^{3}$; in practice, however, this never leads to negative densities. $\mathbf{e}^{3}$ was suggested by Skilling \& Bryan (1984), after some experience with this and other choices. In the search space, we construct quadratic expansions of $S(f)$ and $C(f)$ around the densities $f_{i j m}^{0}$ of the last iteration step

$$
\begin{aligned}
& \tilde{S}(f)=S\left(f^{0}\right)+\sum_{\mu=1}^{3} S_{\mu} x_{\mu}+\frac{1}{2} \sum_{\mu=1}^{3} \sum_{\nu=1}^{3} h_{\mu \nu} x_{\mu} x_{\nu}, \\
& \tilde{C}(f)=C\left(f^{0}\right)+\sum_{\mu=1}^{3} C_{\mu} x_{\mu}+\frac{1}{2} \sum_{\mu=1}^{3} \sum_{\nu=1}^{3} t_{\mu \nu} x_{\mu} x_{\nu},
\end{aligned}
$$

where

$$
f_{i j m}=f_{i j m}^{0}+\sum_{\mu=1}^{3} x_{\mu} e_{i j m}^{\mu} .
$$

Note that the expansion of $C(f)$ in (20) terminates at the second derivative and is therefore exact. The coefficients in (19) and (20) can be calculated from

$$
\begin{aligned}
S_{\mu} & =\sum_{i j m} \frac{\partial S}{\partial f_{i j m}} e_{i j m}^{\mu} \\
h_{\mu \nu} & =\sum_{i j m} \sum_{i^{\prime} j^{\prime} m^{\prime}} e_{i j m}^{\mu} \frac{\partial^{2} S}{\partial f_{i j m} \partial f_{i^{\prime} j^{\prime} m^{\prime}}} e_{i^{\prime} j^{\prime} m^{\prime}}^{\nu} \\
C_{\mu} & =\sum_{i j m} \frac{\partial C}{\partial f_{i j m}} e_{i j m}^{\mu} \\
t_{\mu \nu} & =\sum_{i j m} \sum_{i^{\prime} j^{\prime} m^{\prime}} e_{i j m}^{\mu} \frac{\partial^{2} C}{\partial f_{i j m} \partial f_{i^{\prime} j^{\prime} m^{\prime}}} e_{i^{\prime} j^{\prime} m^{\prime}}^{\nu} .
\end{aligned}
$$

It is at this point that we meet the great advantage of 
(11) over (10) because both $S_{\mu}$ and $h_{\mu \nu}$ can be obtained from very simple expressions:

$$
\begin{gathered}
S_{\mu}=\sum_{i j m}\left(\ln A-\ln f_{i j m}\right) e_{i j m}^{\mu}, \\
h_{\mu \nu}=-\sum_{i j m} e_{i j m}^{\mu} e_{i j m}^{\nu} / f_{i j m} .
\end{gathered}
$$

Calculation of $C_{\mu}$ and $t_{\mu \nu}$ is a bit more involved and we defer its description to Appendix $B$. To simplify the calculations even further, we introduce linear combinations $\mathbf{E}^{\mu}$ of $\mathbf{e}^{1}, \mathbf{e}^{2}$ and $\mathbf{e}^{3}$ such that $h_{\mu \nu}$ becomes minus the identity. According to (24), this can always be done by a Gramm-Schmidt orthogonalization with $\delta_{i j m, i^{\prime} j^{\prime} m^{\prime}} / f_{i j m}$ as metric, In the next step, we diagonalize $t_{\mu \nu}$. Our final model is then

$$
\begin{gathered}
f_{i j m}=f_{i j m}^{0}+\sum_{\mu} y_{\mu} E_{i j m}^{\mu}, \\
\tilde{S}(f)=S\left(f^{0}\right)+\sum_{\mu} \hat{S}_{\mu} y_{\mu}-\frac{1}{2} \sum_{\mu} y_{\mu}^{2}, \\
\tilde{C}(f)=C\left(f^{0}\right)+\sum_{\mu} \hat{C}_{\mu} y_{\mu}+\frac{1}{2} \sum_{\mu} \gamma_{\mu} y_{\mu}^{2},
\end{gathered}
$$

where the $\gamma_{\nu}$ are the eigenvalues of $t_{\mu \nu}$ referred to above and $\hat{S}_{\mu}$ and $\hat{C}_{\mu}$ are the transformed $S_{\mu}$ and $C_{\mu}$. Maximization of $\tilde{Q}(f)=\alpha \tilde{S}(f)-\tilde{C}(f)$ yields

$$
y_{\mu}=\left(\alpha \hat{S}_{\mu}-\hat{C}_{\mu}\right) /\left(\gamma_{\mu}+\alpha\right) .
$$

We still have to choose $\alpha$ such that the constraint of (8) is satisfied. This can most easily be done by choosing $\alpha$ such that $\tilde{C}(f)=M . \quad \alpha$ can be determined easily since the value of $\tilde{C}$ increases monotonically in $\alpha$. Of course, in the first part of the run a situation may occur in which this criterion cannot be satisfied. The minimum value of $\tilde{C}$ that can be obtained at every step is given by

$$
\tilde{C}_{\min }=C\left(f^{0}\right)-\frac{1}{2} \sum_{\mu} \hat{C}_{\mu} \hat{C}_{\mu} / \gamma_{\mu}
$$

The choice of $\alpha$ such that $\tilde{C}(f)=\tilde{C}_{\min }$ would cause the density to be completely determined, at this step, by the constraint and not by the entropy. We therefore choose $\alpha$ such that $\tilde{C}(f)=\tilde{C}_{\text {aim }}$, with

$$
\tilde{C}_{\text {aim }}=\max \left(\frac{2}{3} \tilde{C}_{\min }+\frac{1}{3} C_{0}, M\right) .
$$

If the optimization starts from a flat image, the value of $C(f)$ will gradually decrease during the first part of the run and be constant once the surface $C(f)=$ $M$ has been reached.

Since the quadratic model will be inaccurate at large distances, a limit has to be placed on the difference $\delta f_{i j m}$. Skilling \& Bryan (1984) have chosen

$$
l^{2}(f)=\sum_{i j m} \frac{\left(\delta f_{i j m}\right)^{2}}{f_{i j m}}=\sum_{\nu} y_{\nu}^{2} \leq l_{0}^{2} .
$$

The value of $l_{0}^{2}$ should be $O\left(\sum f\right)$. In all our calculations, a value of $l_{0}^{2}=0.2 \sum f$ was used. So another
Lagrange multiplier is introduced. We have to maximize

$$
\tilde{Q}=\alpha \tilde{S}(f)-\bar{C}(f)-\beta l^{2}(f), \quad \beta \geq 0 .
$$

The maximum of (32) can be found at

$$
y_{\mu}=\left(\alpha \hat{S}_{\mu}-\hat{C}_{\mu}\right) /\left(2 \beta+\gamma_{\mu}+\alpha\right) .
$$

If the optimization starts with $\beta=0$, the value of $\beta$ is increased until the distance constraint is satisfied. This means that, for $\beta \neq 0$, the desired value of $\tilde{C}$ might not be reached.

\section{APPENDIX $B$}

The matrix $t_{\mu \nu}$ can be calculated from

$$
t_{\mu \nu}=\sum_{i j m} \sum_{i i^{\prime} j^{\prime} m^{\prime}} e_{i j m}^{\mu} \frac{\partial^{2} C}{\partial f_{i j m} \partial f_{i^{\prime} j^{\prime} m^{\prime}}} e_{i^{\prime} j^{\prime} m^{\prime}}^{\nu} .
$$

The Hessian of $C$ is not diagonal, so the calculation of $t_{\mu \nu}$ involves a matrix-vector operation. Calculating this matrix-vector product involves of the order of $N^{2}$ operation, where $N$ is the total number of pixels. Since $N$ can be very large $\left[O\left(10^{6}\right)\right]$, this operation is prohibitive. Using

$$
\frac{\partial^{2} C}{\partial f_{i j m} \partial f_{i j^{\prime} m^{\prime}}}=\sum_{h k l} \sum_{h^{\prime} k^{\prime} l^{\prime}} \frac{\partial^{2} C}{\partial F_{h k l} \partial F_{h^{\prime} k^{\prime} l^{\prime}}} \frac{\partial F_{h k l}}{\partial f_{i j m}} \frac{\partial F_{h^{\prime} k^{\prime} l^{\prime}}}{\partial f_{i^{\prime} j^{\prime} m^{\prime}}},
$$

we may write

$$
\begin{aligned}
t_{\mu \nu}= & \sum_{i j m} \sum_{i^{\prime} j^{\prime} m^{\prime}} e_{i j m}^{\mu} \\
& \times \sum_{h k l} \sum_{h^{\prime} k^{\prime} l^{\prime}} \frac{\partial^{2} C}{\partial F_{h k l} \partial F_{h^{\prime} k^{\prime} l^{\prime}}} \frac{\partial F_{h k l}}{\partial f_{i j m}} \frac{\partial F_{h^{\prime} k^{\prime} l^{\prime}}}{\partial f_{i^{\prime} j^{\prime} m^{\prime}}} e_{i^{\prime} j^{\prime} m^{\prime}}^{\nu}
\end{aligned}
$$

Changing the order of summation, we get

$$
\begin{aligned}
t_{\mu \nu}= & \sum_{h k l} \sum_{h^{\prime} k^{\prime} l^{\prime}} \frac{\partial^{2} C}{\partial F_{h k l} \partial F_{h^{\prime} k^{\prime} l^{\prime}}}\left(\sum_{i j m} e_{i j m}^{\mu} \frac{\partial F_{h k l}}{\partial f_{i j m}}\right) \\
& \times\left(\sum_{i^{\prime} j^{\prime} m^{\prime}} e_{i^{\prime} j^{\prime} m^{\prime}}^{\nu} \frac{\partial F_{h^{\prime} k^{\prime} l^{\prime}}}{\partial f_{i^{\prime} j^{\prime} m^{\prime}}}\right) \\
= & \sum_{h k l} \sum_{h^{\prime} k^{\prime} l^{\prime}} \frac{\partial^{2} C}{\partial F_{h k l} \partial F_{h^{\prime} k^{\prime} l^{\prime}}}, \bar{J}\left(e^{\mu}\right)_{h k l^{\prime}}, \bar{f}\left(e^{\nu}\right)_{h^{\prime} k^{\prime} l^{\prime}} .
\end{aligned}
$$

The symbol 7 expresses a (three-dimensional) Fourier transform. The matrix $\partial^{2} C / \partial F_{h k l} \partial F_{h^{\prime} k^{\prime} l}$ is diagonal since

$$
\begin{aligned}
& \frac{\partial^{2} C}{\partial F_{h k^{\prime}} \partial F_{h^{\prime} k^{\prime} l^{\prime}}}=0 \quad \text { for } h k l \neq h^{\prime} k^{\prime} l^{\prime}, \\
& \frac{\partial^{2} C}{\partial F_{h k l}^{2}}=\frac{2}{\sigma_{h k l}^{2}} \text { for } h k l=h^{\prime} k^{\prime} l^{\prime} .
\end{aligned}
$$

Then,

$$
t_{\mu \nu}=\sum_{h k l}\left(2 / \sigma_{h k l}^{2}\right) \not\left(e^{\mu}\right)_{h k l} \not\left(e^{\nu}\right)_{h k l}
$$


So $t_{\mu \nu}$ can be calculated using the fast Fourier transform, which involves $O\left(N \log _{2} N\right)$ operations.

The vectors $\partial C / \partial f_{i j m}$ and $\mathbf{e}^{3}$ can be calculated in an analogous way. In total, six Fourier transforms are need in each cycle:

(i) one Fourier transform for the determination of $F_{h k l}$ from the current EDD;

(ii) one Fourier transform for the determination of the vector $\partial C / \partial f_{i j m}$;

(iii) one Fourier transform for the determination of the vector $\mathbf{e}^{3}$;

(iv) three Fourier transforms for the determination of $t_{\mu \nu}$.

\section{References}

Baert, F., Coppens, P., Stevens, E. D. \& Devos, L. (1982). Acta Cryst. A38, 143-151.
Bruning, H. \& Feil, D. (1992). Acta Cryst. A48, 865-872.

Craven, B. M., Weber, H. P. \& He, X. (1987). Technical Report TR-87-2. Department of Crystallography, Univ. of Pittsburgh, PA 15260 , USA.

Eriksson, A. \& Hermansson, K. (1983). Acta Cryst. B39, 703-711.

Hansen, N. K. \& \& Coppens, P. (1978). Acta Cryst. A34, 909921.

HirshFeld, F. L. (1971). Acta Cryst. B27, 769-781.

HiRSHFELD, F. L. (1977). Isr. J. Chem. 16, 226-229.

Jauch, W. \& Palmer, A. (1993). Acta Cryst. A49, 590-591.

JAYNES, E. T. (1983). (Collected works.) In Papers on Probability, Statistics and Statistical Physics, edited by R. D. Rosenkrantz. Dordrecht: Reidel.

LeComTe, C. (1991). In The Application of Charge Density Research to Chemistry and Drug Design, edited by G. A. JefFrey \& J. F. Piniella. New York: Plenum.

SaKa, T. \& Kato, N. (1986). Acta Cryst. A42, 469-478.

SaKata, M. \& SATo, M. (1990). Acta Cryst. A46, 263-270.

Skilling J. \& Bryan, R. K. (1984). Mon. Not. R. Astron. Soc. 211, 111-124.

Acta Cryst. (1994). A50, 391-402

\title{
Solution Scattering from Biopolymers: Advanced Contrast-Variation Data Analysis
}

\author{
BY D. I. SVERGUN* \\ European Molecular Biology Laboratory, Hamburg Outstation, Notkestrasse 85, D-22603 Hamburg, \\ Germany
}

(Received 30 September 1993; accepted 1 December 1993)

\begin{abstract}
New approaches to the interpretation of contrastvariation data from monodisperse systems using spherical harmonics are presented. A general method is given for evaluating the particle shape and internal structure and expressions for the scattering from the density fluctuations inside a particle with a known shape are derived. Further, the scattering from twocomponent particles is analyzed in terms of the positions and/or shapes of the components and the information content of the contrast-variation data is discussed. The methods can be used for advanced low-resolution structure analysis of various types of biopolymers in solution.
\end{abstract}

\section{Introduction}

Small-angle scattering (SAS) is one of the most effective methods of investigating the superatomic structure of native biopolymers and their complexes

\footnotetext{
* On leave from the Institute of Crystallography, Russian Academy of Sciences, Leninsky prospekt 59, 117333 Moscow, Russia.

in solution (Feigin \& Svergun, 1987). The SAS intensity $I(s)$ of a dilute monodisperse solution of biopolymers is proportional to the scattering from a single particle averaged over all orientations [here $s$ denotes the modulus of the scattering vector $\mathbf{s}, s=$ $(4 \pi / \lambda) \sin \theta, \lambda$ is the wavelength and $2 \theta$ is the scattering angle]. Recent developments in experimental techniques (see e.g. Feigin \& Svergun, 1987, ch. 8; Koch, 1991) allow one to register precise SAS curves over a wide range of scattering vectors and comprehensive data interpretation is therefore of great importance.

As solution scattering provides low-resolution structural information, it is usually interpreted in terms of homogeneous particles. Shape modeling is still one of the most frequently used approaches (Feigin \& Svergun, 1987, ch. 3.5). The direct method of Stuhrmann (1970b) with the recent improvements of Svergun \& Stuhrmann (1991) allows lowresolution shape determination using a straightforward procedure. As the scattering-length density of biopolymers in solution is by no means homogeneous, shape modeling or determination, in a strict sense, has to be applied to the 'shape scattering' 